\title{
CONTINENTAL LITHOSPHERE DEEP STRUCTURE RESEARCHES ON THE BASE OF SCIENTIFIC DEEP DRILLING
}

\author{
Kouznetsova E.I., Galdin N.E.
}

(Research Industrial Company GERS, Apt. 62, d.4, 2-nd Pereulok Truzhennikov, 119121 Moscow Russia)

Investigation of deep structure and geodynamic regimes of the lithosphere on the base drilling and geotransects are extreamely important for fundamental Earth sciences development and promotion of the applied problems decision. Especially it's important for understanding of processes of oil, gas and ore generation, evaluation of geothermal energy, integration of petrological, geological, geophysical data and kimberlite volcanism.

At present time the next scientific deep and superdeep boreholes are drilled in Russia: Kola SD-3 (12261 m), Vorotilov (5374 m), Ural (4950 m), Tyrnyaz (4001 m), Colva (7057 m), Tyman-Pechora $(6904 \mathrm{~m})$, Tumen $(7500 \mathrm{~m})$, Kuban $(4000 \mathrm{~m})$, Tujmazinskaya (3845 $\mathrm{m})$, Minnibaevskay $(5099 \mathrm{~m})$, Novoyelkhovskaya-20009 $(5500 \mathrm{~m})$. The drilling is continuing now in Novoyelkhovskaya-20009, Ural and Tuman wells. The Kola superdeep borehole works as geolaboratory and deep observatory for long-term geological and geophysical investigations.

Kola and Novoyelkhovskaya boreholes are of the greatest scientific interest from point of view of their geologocal-geophysical logs comparison due to their positions at the opposite parts of the Russian Platform (eastern part of the Baltic Shield and South-eastern part of the Russian Plate). They are well investigated by geological-geophysical methods. The logs of Novoyelkhovskaya borehole correlate well with geological-geophysical profiles along geotransects Granite and Quartz.

The investigations of stress state and geothermal and fluid regimes of the lithosphere were done on the base of Kola and Novoyelkhovskaya superdeep boreholes. The crust structure in the region of the Kola superdeep borehole is characterized by existance of intracrustal waveguides - zones of increased mobility, substance fluidity and higher permeability. Convective movements of fluid could arise in waveguides under special parameters of medium. It provides the step character of heat flow variations. Vertical variations of heat flow are explained by many factors: paleoclimate, fluid filtration, heat properties of rocks, stress state, history of evolution.

The main directions of researches were: 1) geological-geophysical investigations along geotransects in the region of the Kola superdeep borehole; 2) geological-geophysical investigations in the Kola well SD-3; 3) thermomechanical simulation of the Baltic Shield geological structures formation and evolution and the geothermal regime modelling.

Specific form of Pechenga structure gives unique possibility to compare geologicalgeophysical properties of the same geological layers at the Earth's surface and at the depth.

There are some specific geological structures - overthrusts and overlaps at the Baltic Shield. It is possibilite to get analytical decisions of thermomechanical equations for such structures evolution. Complex investigations on the base of geological-geophysical data and mathematical modelling could be a clue for the Earth crust origin problem decision.

Seismic investigations weve carried out by method of vertical seismic profiling during long period of time (1970-1989) with the purpose of studying: velocity and damping of elastic waves in he vertical section of borehole SD-3, complex wave field, nature of seismic boundaries in the upper part of the earth crust (in particular subhorizon boundaries registered by DSS method (Depp Seismic Sounding) at the depth of $5-7 \mathrm{~km}$ and $9-13 \mathrm{~km}$ ). At the first and second stages of drilling six points of 
expositions were used spaced $0.15-3 \mathrm{~km}$ from SD-3 collar, at the third stage - two points spaced 0.68 and $2.96 \mathrm{~km}$ from the collar. Observation pitch constituted $20 \mathrm{~m}$, seismic stations - PoiskSK, VSP-1, seismic receivers of SVM-30 and SV1-20TS types were used. Vertical component of oscillation was mainly registered in limited range of depth, in the upper part of borehole SD-3 three-component observations were carried out. The investigations have been conducted by methods of vertical seismic profiling.

As a result diagram of layer velocities of longitudinal waves for SD-3 section was constructed up to depth of $12 \mathrm{~km}$. Minimum thickness of layers was $0,2 \mathrm{~km}$, error for velocity determination $V_{p}=0,1 \mathrm{~km} / \mathrm{s}$. Velocity of transversal waves was studied with less grade of reliability because $\mathrm{S}$-waves registration in subsequent coming and connected with it difficulties while correlating the waves. Above-mentioned velocity section was utilised for correlation of sections from different shafts of borehole SD-3 and for studying the nature of seismic boundaries. In particular, discovered by DSS method subhorizon seismic boundary with the velocity of $6.6 \mathrm{~km} / \mathrm{s}$ (surface of "basalt layer") was not confirmed on vertical velocity section.

Investigations of wave field by vertical seismic profiling showed the presence of clearly defined straight longitudinal and transversal waves through the whole lenght of vertical profile. Those waves form passing exchange waves of two types: PS and SP.

Reflections correspond mainly to monotype longitudinal waves and in general were poorly defind, their correlation was extremely difficult and beared "dash"character (similar to wave fields MOV). Analysis of wave field by method of vertical seismic profiling received when utilising rather remote point of explosion (about $40 \mathrm{~km}$ ) showed that the matter concerned not seismic boundaries of the 1st type as it was supposed before but complex waveguiding structures.

On the base of geological-geophysical researches it was determined that region of the Kola well SD-3 is connected with extended active tectonic zone of high permeability. Tectonic activity and fluid mobility there decrease with depth.

This anomalous zone is characterized by lower seismic velocity (waveguide). The investigations show that this zone is of higher jointing, secondary, partial breaking and destruction of rocks at the depth of 9.2-11.2 km in Archaean complex (7-12 km). This zone of deep crystal rocks (gneisses, granites, amphybolites et al.) is characterized by rough increase of porosity, permeability and content of secondary minerals. Core of this zone is represented by disks and plated due to intensive subhorizontal jointing. On the base of deep geological structure investigations of eastern part of Baltic shield it is possible to consider this zone as the zone with specific mechanical properties and structure. The slow waterexchange zone at the depth of $2.5-4.5 \mathrm{~km}$ was considered as waveguide too. Below this zone the zone of stagnant water of chloride-calcium and chloride-natrium content of high mineralization is situated. Thus SD-3 crossection investigations gave possibility to determine the high permeability zones at different depth, where zones of kimberlite may be development. 